\title{
Determinación de IgG contra Chlamidya trachomatis en mujeres con artritis de la Ciudad de Bogotá D.C. Un estudio piloto
}

\author{
Determination of IgG in women infected with chlamydia trachomatis and \\ reactive- arthritis from bogota, colombia: a pilot study
}

Alejandra Mantilla ${ }^{2}$, Jhonathan Martínez ${ }^{1}$, Mateo Santiago Ramírez ${ }^{1}$ Luis Felipe Olave ${ }^{1}$, Adriana Paola Jutinico Shubach³ ${ }^{3}$ María C. Gómez², Ruth Mélida Sánchez Mora²

\section{Resumen}

Objetivo. Evaluar la presencia de IgG contra C. trachomatis en mujeres entre 18 a 30 años con diagnóstico de artritis de la ciudad de Bogotá D.C. Método. Los grupos de estudio están compuestos por un grupo de casos AR (mujeres con diagnóstico de artritis reumatoide y reactiva) y un grupo control. Se obtuvieron muestras de suero y se realizó la determinación cuantitativa de IgG contra C. trachomatis, la determinación PCR (proteína C reactiva) y FR (factor reumatoide). Resultados. Las variables de talla y peso descritas en la población de estudio, muestran un comportamiento homogéneo, por lo cual no interfieren en los resultados obtenidos. Las medias se compararon por los test Mann Whitney y $t$ test no pareado. El porcentaje de resultados positivos en la determinación cualitativa de PCR fueron de: 36,6\% para el grupo de casos y 14,5\% para el grupo control. En la determinación cualitativa de FR el porcentaje de resultados positivos fueron: 48,8\% para el grupo de casos y $5,3 \%$ para el grupo control. Finalmente, el porcentaje de resultados positivos en la determinación de anticuerpos $\operatorname{IgG}$ específicos contra $C$ trachomatis fue de $2,4 \%$ para el grupo de casos y 22,4\% para el grupo de controles. Las variables de PCR y FR mostraron mayor frecuencia de resultados positivos en el grupo de casos, en comparación con el grupo control, lo cual se correlaciona con la existencia de procesos inflamatorios propios del desarrollo de artritis. Sin embargo el grupo control presentó mayor frecuencia de anticuerpos IgG específicos contra $C$. trachomatis en comparación con el grupo de casos. Estos resultados hacen necesario evaluar a futuro, el comportamiento de las pacientes del grupo control con resultados positivos de $\operatorname{IgG}$ contra $C$. trichomatis que permitirían observar una posible aparición de síntomas relacionados con artritis.

Palabras clave: C.trachomatis, IgG, artritis, PCR, FR.

\section{Abstract}

Objective. Evaluate the presence of $\operatorname{IgG}$ in women infected with $C$. trachomatis, and diagnosed with arthritis, aged 18-30 years, in Bogota, Colombia. Methods. This project is composed of two study groups: Firstly, female cases diagnosed with rheumatoid reactive-arthritis and secondly, a control

\footnotetext{
${ }^{1}$ Estudiantes Universidad Colegio Mayor de Cundinamarca. Bogotá, Colombia.

2Docentes Universidad Colegio Mayor de Cundinamarca Bogotá, Colombia.

${ }^{3}$ Joven Investigadora Colciencias Universidad Colegio Mayor de Cundinamarca. Bogotá, Colombia

Correspondencia: amantilla@unicolmayor.edu.co

Recibido: 13-02-2016 Aceptado: 17-04-2016
} 
group. Quantitative determination of $\mathrm{IgG}$ in serum samples from patients with $C$. trachomatis. Were determined also, PCR determination (C reactive protein) and RF (rheumatoid factor) were performed. The statistical analysis included the comparison of means, using Mann Whitney and unpaired test. Results. Patient samples that were positive by PCR: $36.6 \%$ for the female case group, and $14.5 \%$ for the control group. In the qualitative determination of RF positives, results were: $48.8 \%$ for the case group and 5.3\% for the control group. Finally, the percentages of positive results for IgG antibodies determination against $C$. trachomatis were: $2.4 \%$ for the case group and $22.4 \%$ for the control group. PCR and FR variables showed higher frequency of positive results in the female case group compared with the control group, which correlates with the presence of inflammatory processes, where it is typical in the development of arthritis. However, the control group had increased frequency of specific IgG antibodies against $C$. trachomatis, when it is compared with the case female group. These results need to be evaluated in the future, where, the control group that tested positive for IgG against $C$. Trachomatis, would allow to observe the possible apparition of symptoms related to arthritis.

Key Words: C. trachomatis, IgG, arthritis, PCR, FR.

\section{Introducción}

Chlamydia trachomatis (C. trachomatis) es el patógeno más prevalente en las enfermedades de transmisión sexual (ETS) en el mundo occidental. Se considera que el 14\% de casos de mujeres infectadas con esta bacteria son asintomáticos, siendo la enfermedad pélvica inflamatoria (EPI) una de las principales manifestaciones clínicas. Por otro lado etiología de las enfermedades autoinmunes relacionadas con la artritis no se ha esclarecido totalmente, sin embargo, en estudios previos han estudiado la hipótesis que individuos con HLA-B27, podrían presentar fenómeno de reactividad cruzada pos infección con C. trachomatis y Yersinia enterocilitica.

Chlamydia trachomatis es un Bacilo Gram negativo, parásito intracelular obligado. En Estados Unidos, C. trachomatis se ha reportado como una de las enfermedades de trasmisión sexual de mayor prevalencia. Una de las principales síntomas de las infecciones causadas por esta bacteria es la EPI, sin embargo es importante destacar que esta patología cursa de una manera asintomática en un $14 \%$ de la población femenina (1-2).
La artritis reumatoide (AR) se considera como una enfermedad autoinmune que generalmente se manifiesta en personas que están en edad productiva y se caracteriza por ser degenerativa, crónica y multisistémica, focalizada específicamente en la membrana sinovial, que es la encargada de producir el líquido que lubrica las articulaciones para que pueda haber movimiento sin desgaste óseo ni dolor. Al haber daño de dicha membrana productora del líquido sinovial por la respuesta inmunológica, la producción de éste irá disminuyendo, lo que a su vez permite que el desgaste óseo sea evidente por medio de patologías como malformaciones en las articulaciones. Ésta enfermedad está asociada con mayor frecuencia a mujeres que a hombres en una relación 3:1 respectivamente a nivel mjundial. Su prevalencia en Latinoamérica oscila en $0,5 \%$, con incidencia en mujeres de 40 a 60 años de edad (2-3). Debido a la cronicidad y al daño físico ocasionado en las personas que padecen de artritis reumatoide, se observa afectada la calidad de vida al tener limitaciones físicas y astenia, las personas entran en conflictos psicológicos al depender de otras para ejercer actividades cotidianas (4-5). 
Por otro lado, la artritis reactiva es una enfermedad que genera inflamación en las articulaciones, luego de un proceso infeccioso generalmente genitourinaria o gastrointestinal acompañado de lesiones mucocutáneas y artritis, la cual se considera la triada de artritis reactiva (6). La forma venérea se ha asociado con mayor frecuencia con Chlamydia trachomatis y la formas disentéricas se han asociado a Salmonella, Shiguella, Yersinia o Campilobacter por consumo de alimentos contaminados (1). La artritis reactiva asociada a infección en vías urinarias se presenta con mayor frecuencia en individuos entre 20 y 40 ańos de edad con vida sexual activa (7). La artritis reactiva desarrollada post-infección disentérica o gastrointestinal es más frecuente en niños. En estudios previos realizados en un Hospital de Tailandia, se describió la media de edad de 23 años para la aparición de la enfermedad. (8).

La etiología de las enfermedades autoinmunes relacionadas con la artritis no se ha esclarecido totalmente. Sin embargo, se ha reportado entre el 40-60\% de los pacientes con este diagnóstico expresan HLA-B27 y HLA-B51. En estudios previos se ha estudiado la hipótesis que individuos con HLA-B27, podrían presentar fenómeno de reactividad cruzada pos infección con $C$. trachomatis y Yersinia enterocolitica.

Se desconoce la prevalencia de la infección por $C$. trachomatis en pacientes con artritis en la ciudad de Bogotá. El presente estudio piloto, busca evaluar la presencia de IgG contra $C$. trachomatis y en pacientes con artritis en un grupo de mujeres de la ciudad de Bogotá D.C.

\section{Materiales y métodos}

Se realizó un estudio observacional, analítico, descriptivo de corte transversal, compuesto por un grupo de casos (41 mujeres no relacionadas con diagnóstico de artritis reumatoide o reactiva entre las edades 18 a 30 ańos) y uno control (76 mujeres no relacionadas entre las edades 18 a 30 años sin diagnóstico de artritis ni enfermedades de base como diabetes, anemias, tuberculosis, enfermedades autoinmunes entre otras). Se excluyeron del estudio pacientes que estaban en tratamiento con medicamentos en el momento de la toma de muestra. El estudio se realizó en el laboratorio de Biotecnología y Genética UCMC en las instalaciones de la Universidad de Colegio Mayor de Cundinamarca sede Bogotá D.C.

Población de estudio. La población está compuesta por mujeres residentes de la ciudad de Bogotá, quienes firmaron un consentimiento informado y decidieron participar libremente en el estudio. Las muestras de suero se tomaron por personal entrenado, se procesaron y conservaron en los laboratorios de la Universidad Colegio Mayor de Cundinamarca.

Toma de muestra. Las muestras se obtuvieron por venopunción por la técnica de tubo al vacío en tubos tapa roja, para obtener el suero por centrifugación de la muestra a 3.000 r.p.m. durante 10 minutos.

Pruebas serológicas. La determinación de anticuerpos se realizó con el kit de ELISA de determinación IgG contra $C$. trachomatis de la casa comercial DIA. PRO. Brevemente, los micropocillos están cubiertos con péptidos específicos derivados del antígeno MOMP de $C$. trachomatis. La determinación de proteína $\mathrm{C}$ reactiva PCR y la determinación de factor reumatoide FR se realizó con el kit de aglutinación de BioSystems. Para la determinación de IgG contra $C$. trachomatis se utilizó el lavador de microplacas MW 12A y el lector MR- 96.

Análisis estadístico. La descripción de la sensibilización de $C$. trachomatis se realizó con estadística descriptiva, utilizando el programa Graph Pad Prims 5,0 para la comparación de medias y las pruebas de normalidad.

\section{Resultados}

En el estudio participaron dos grupos un grupo caso (41 mujeres) y un grupo control (76 mujeres). Los grupos de casos estaban conformados por pacientes con diagnóstico médico proporcionado por sus 
respectivas E.P.S. tanto de artritis reumatoide (22 mujeres) como de artritis reactiva (19 mujeres). El grupo control (76 mujeres) fueron personas no relacionadas que decidieron participar en el estudio de manera voluntaria las cuales no tienen diagnóstico de artritis, enfermedades autoinmunes u otra enfermedad crónica.

\section{Descripción de la población}

Los 2 grupos (artritis y grupo control), se analizaron bajo el programa de Graph Pad prims 5.0 donde se determinó la media, la desviación estándar, los percentiles y la normalidad de las siguientes variables: edad, talla y peso. Las variables se compararon por los tests de comparación Mann Whithey y t test no pareado.

La media de edad de los grupos analizados se puede observar en la Tabla 1. La media del grupo casos fue de 23,88 años y del grupo control fue de 21,46 años, el test de normalidad de Kolmogorov-Smirnov demostró que la variable de edad tiene una distribución no normal. El test Mann
Whithey demostró una diferencia estadísticamente significativa al comparar los grupos de casos vs el grupo control.

En la Tabla 1 se observan la media del peso de los grupos analizados. Se evidencia una media de peso en el grupo de casos de 59,71 Kg y en el grupo control de $58.18 \mathrm{Kg}$. El test de normalidad de KolmogorovSmirnov nos muestra que la población tiene una distribución de peso no normal. Además se realizó el test Mann Whitney, en el cual no se observó diferencia estadística al comparar los grupos del estudio. Éstos resultados evidencian que la variable de peso es homogénea en los grupos de estudio.

La media de la talla de los grupos analizados se puede observar en la Tabla 1 . Se evidencia una media de talla en el grupo de casos es de 1,615 m, y en el grupo control de $1.59 \mathrm{~m}$. El test de normalidad de Kolmogorov-Smirnov nos muestra que la distribución de talla es normal. Al realizar el test t no pareado se observó que no hay diferencia estadísticamente significativa en los grupos de estudio.

Tabla 1. Descripción de la población de estudio. KS: Kolmogórov-Smirnov prueba de normalidad SW: Shapiro-Wilk prueba de normalidad Valor de $\mathrm{p}<0,05$ fueron estadísticamente significativos.

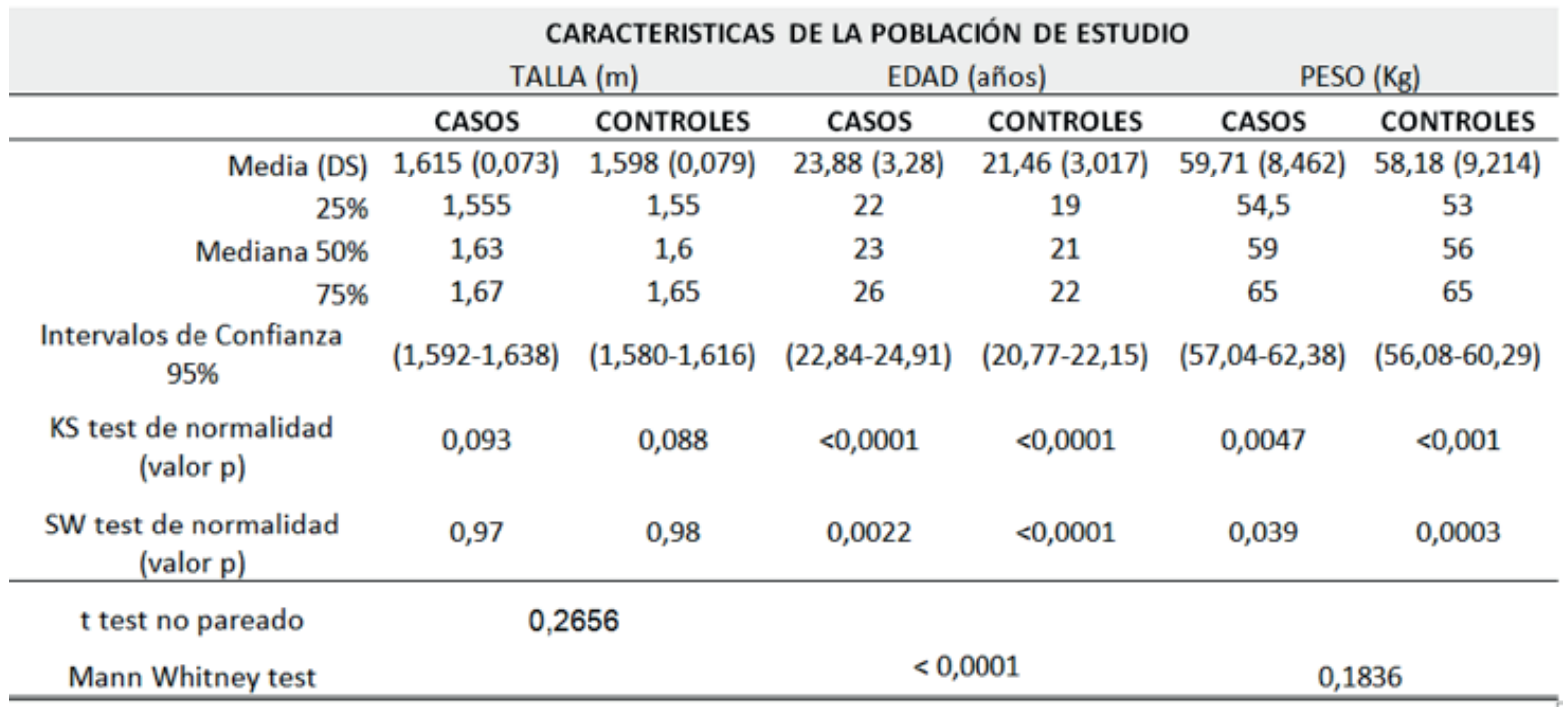

\section{Determinación Cualitativa De Proteina C Reactiva (PCR)}

Se realizó la determinación cualitativa de Los resultados obtenidos en éste ensayo la proteína $\mathrm{C}$ reactiva en los sueros de los fueron determinados de acuerdo al inserto de pacientes presentes en los grupos de estudio. la casa comercial BioSystems, el cual permite 
determinar la presencia de proteína $\mathrm{C}$ reactiva en suero, como marcador de inflamación, donde se consideran positivas las muestras que evidencien aglutinación (mayor o igual a $6 \mathrm{mg} / \mathrm{L})$ y en las negativas no se evidencia aglutinación (menor 6mg/L). Figura 1A.
Para el grupo de casos el $63,4 \%$ de la población presento PCR negativos y el 36,6\% presentó resultados positivos. En el grupo control, el 14,5\% de la población presentó presencia en suero de PCR positivo y el $85,5 \%$ de la población tenía niveles séricos negativos. Figura $1 \mathrm{~B}$ y $1 \mathrm{C}$.
A.

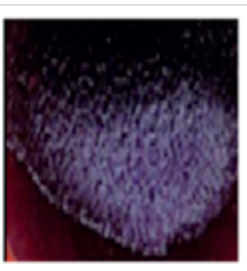

POSITIVO

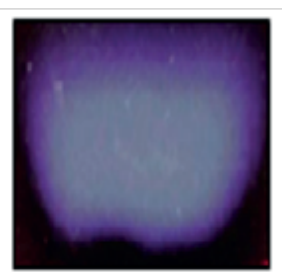

NEGATIVO
B.

\begin{tabular}{ccc}
\hline & CASOS & CONTROLES \\
\hline POSITIVO & $15(36,6 \%)$ & $11(14,5 \%)$ \\
NEGATIVO & $26(63,4 \%)$ & $65(85,5 \%)$ \\
\hline N & $41(100 \%)$ & $76(100 \%)$
\end{tabular}

\section{Determinación de PCR por técnica de aglutinación}

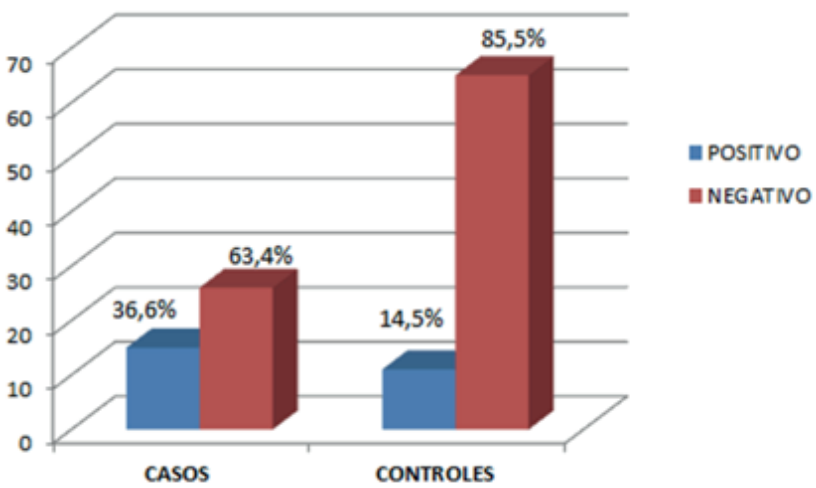

Figura 1. Resultados de PCR por la técnica de aglutinación. A. Imágenes de una prueba positiva y negativa para la técnica de PCR por aglutinación. B. Tabla donde se presentan resultados en frecuencia y porcentaje para la técnica de PCR en los dos grupos de estudio. C. Diagrama de barras, resultados en porcentaje para la técnica de PCR en los dos grupos de estudio.

\section{Determinación cualitativa de Factor Reumatoide (FR)}

Se realizó la determinación cualitativa del factor reumatoide. El kit de BioSystems considera un resultado negativo valores séricos $<30 \mathrm{Ul} / \mathrm{mL}$ y como resultado positivo valores séricos $>30 \mathrm{Ul} / \mathrm{mL}$.

En el grupo de mujeres con diagnóstico de artritis reumatoide o reactiva se observó que el 48,8\% (20 mujeres) fueron positivas y $51,2 \%$ fueron negativas (21 mujeres). En el grupo control se observó que el $5,3 \%$ (4 mujeres) fueron positivas y el $94,7 \%$ (72 mujeres) fueron negativas. Los resultados se observan en la Figura 2A y 2B.
A.

\begin{tabular}{ccc}
\hline & CASOS & CONTROLES \\
\hline POSITIVOS & $20(48,8 \%)$ & $4(5,3 \%)$ \\
NEGATIVOS & $21(51,2 \%)$ & $72(94,7 \%)$ \\
N & $41(100 \%)$ & $76(100 \%)$ \\
\hline
\end{tabular}

B. Determinación de FR por la técnica de aglutinación

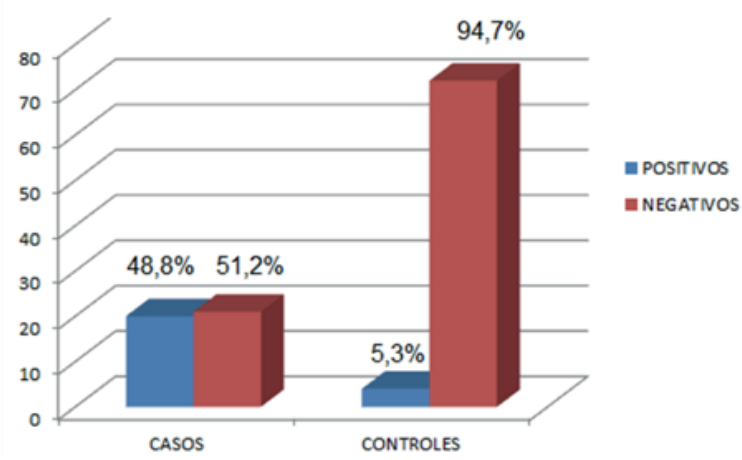

Figura 2. Resultados de FR por la técnica de aglutinación. A. Tabla donde se muestran las frecuencias y los porcentajes para la técnica de PCR en los dos grupos de estudio. B. Diagrama de barras, resultados en porcentaje para la técnica de PCR en los dos grupos de estudio. 


\section{Determinación de anticuerpos IgG específicos contra C. trachomatis}

Para la validación de los resultados se siguieron las indicaciones del inserto del kit de Elisa Dia Pro diagnostic Bioprobes. La interpretación de los resultados según el kit establece que las muestras con concentraciones inferiores a $5 \mathrm{arbU} / \mathrm{mL}$ son consideradas negativas y las muestras con concentraciones superiores a $5 \mathrm{arbU} / \mathrm{mL}$ son consideradas positivas, para anticuerpos IgG específicos contra $C$. trachomatis, cada muestra se realizó por duplicado. Figura 3A.
Se observó la concentración de anticuerpos $\operatorname{IgG}$ contra C. trachomatis en el grupo casos, el 97,6\% de los pacientes presentaron una concentración $<5 \mathrm{arbU} / \mathrm{mL}$, es decir, que se consideran negativas para anticuerpos IgG contra C. trachomatis y un $2,4 \%$ de los pacientes fueron positivos. En el grupo control el 77,64\% de los pacientes presentaron una concentración $<5 \mathrm{arbU} / \mathrm{mL}$ (negativos) y el $22.4 \%$ de los pacientes presentaron una concentración $>5 \mathrm{arbU} / \mathrm{mL}$ (positivos). Los resultados de la concentración de anticuerpos IgG específicos contra $C$. trachomatis en los grupos de estudio se observa en la Figura 3B y 3C.
A.

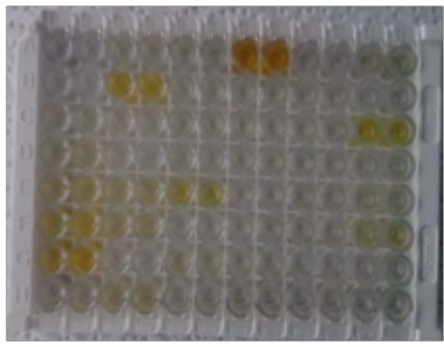

B.

\begin{tabular}{ccc}
\hline & CASOS & CONTROLES \\
\hline POSITIVO & $1(2,4 \%)$ & $17(22,4 \%)$ \\
NEGATIVO & $40(97,6 \%)$ & $59(77,6 \%)$ \\
\hline N & $41(100 \%)$ & $76(100 \%)$
\end{tabular}

C. Determinación de lgG a C. trachomatis por la técnica de ELISA

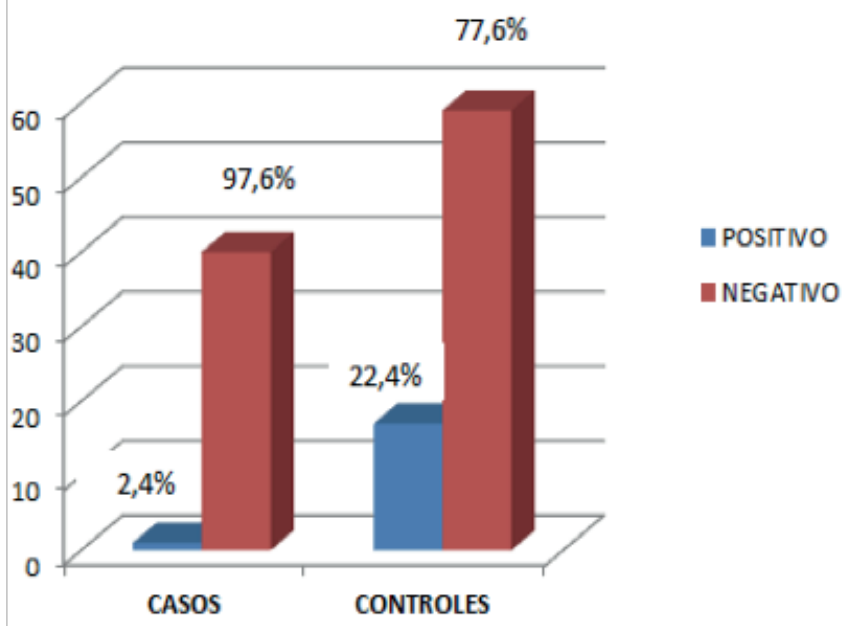

Figura 3. Resultados de IgG contra C. trachomatis por la técnica de ELISA. A. Imágenes de la técnica de ELISA para la determinación de IgG a C. trachomatis. B. Tabla donde se reportan los resultados en frecuencia y porcentaje para la técnica de ELISA en los dos grupos de estudio. C. Diagrama de barras, resultados en porcentaje para la técnica de ELISA en los dos grupos de estudio.

\section{Discusión}

El desarrollo de artritis asociado por una infección genitourinaria previa con $C$. trachomatis, es una patología cuya etiología no se ha esclarecido totalmente. A nivel nacional son pocas las publicaciones y se desconoce su prevalencia e incidencia en la población nacional. Según Molina et al., en el 2011 la prevalencia de la enfermedad se presenta con mayor frecuencia en hombres entre los 20 y 40 años de edad (7). Aunque un estudio realizado por Zarco Montejo P. et al., en el 2011 afirma que el $75 \%$ de la población femenina infectada por $C$. trachomatis es asintomática, por lo cual no son diagnosticadas ni tratadas a tiempo (9). La población en el estudio piloto fue de 41 mujeres con artritis entre los 18 y 30 ańos de edad. Se tomó esta población puesto que al no ser diagnosticadas, ni tratadas en una etapa temprana de la enfermedad, esta población es vulnerable a presentar un tipo de artritis como lo es la artritis reactiva o Síndrome de Reiter. 
En la población de estudio la media de edad en el grupo casos fue de 23,88 ańos y la del grupo control fue de 21,46 años de edad, estos resultados se encuentran relacionados con el rango de edades en que se presenta la enfermedad, según lo reportado por Arraiz $N$ et al., en el 2008 (10). La media de talla para la población de casos fue de $1,615 \mathrm{~m}$, no se observó diferencia significativa con el grupo control. La media de peso para el grupo de casos fue de $59,71 \mathrm{Kg}$ y para el grupo control de $58,18 \mathrm{Kg}$. La población estudiada, en cuanto a talla y peso es homogénea y la diferencia no fue estadísticamente significativa.

El porcentaje de pacientes positivos para la proteína C- Reactiva en el grupo de casos fue de $36,6 \%$ y de $14,5 \%$ en pacientes controles. La proteína C- reactiva no es una prueba diagnóstica para la enfermedad, sin embargo es un indicador cualitativo, que el paciente está cursando por un proceso inflamatorio. En este estudio las pacientes que cursan con artritis presentan resultados positivos para PCR asociada a la respuesta inmunológica focalizada en las articulaciones (11). En el estudio realizado por Molina Restrepo en el 2007 se determina que la PCR puede estar aumentada en personas obesas o que estén ingiriendo anticonceptivos orales o que estén cursando un proceso infeccioso, por lo cual se determina que los pacientes positivos en el grupo control pueden estar relacionados con alguna de las anteriores causas (12).

Otro análisis de laboratorio realizado en éste estudio fue el factor reumatoide donde el porcentaje de resultados positivos para el grupo de casos fue de $48,8 \%$.

Según el estudio realizado por Molina Restrepo en el 2007 describe que la prueba del factor rematoide es una prueba de ayuda diagnóstica para las personas con artritis reumatoide juvenil, diferenciándolos de las poblaciones con artritis y artritis reactiva. Sin embargo, es necesario implementar estudios de tipo cohorte que permitan evaluar el comportamiento de estas variables en el tiempo junto al desarrollo y seguimiento de enfermedades con artritis y artritis reactiva. En cuanto al grupo control, un estudio de tipo cohorte permitiría evaluar a aquellos individuos que reflejan el factor reumatoide positivo y si podrían estar predispuestos a desarrollar artritis reumatoide y otra enfermedad inflamatoria crónica o autoinmune a futuro (12). Los pacientes con artritis con resultados positivos de factor reumatoide no están asociados al desarrollo de la enfermedad por previa infección con $C$. trachomatis, pero sí podrían estar asociados a otros microorganismos causantes de infecciones gastrointestinales o co-infecciones (13).

En la detección de anticuerpos $\operatorname{IgG}$ contra $C$. trachomatis se analizó la frecuencia de resultados positivos en los dos grupos de estudio, para el grupo de casos se observó solo 2,4\% de mujeres positivas a IgG contra C. trachomatis.

Se evidencia que el grupo que presenta una mayor prevalencia en la concentración de anticuerpos IgG contra $C$. trachomatis es el grupo control con un $22,4 \%$, lo que indica que en este grupo hay pacientes que cursaron con la infección por C. trachomatis y se consideran una población vulnerable para desarrollar artritis reactiva, como menciona Zarco Montejo et al., en el 2011, el cual explica que en el 75\% las mujeres que presentan artritis reactiva asociada a una previa infección por C. trachomatis son asintomáticas, por lo que este estudio abre las puertas para evaluar más detalladamente al grupo control e incentivar a las mujeres de éste grupo a que tengan un chequeo médico y exámenes confirmatorios para prever el desarrollo de la artritis (8).

En Colombia según un estudio realizado Paredes MC., et al. en el 2015 se afirma que la prevalencia de enfermedades por transmisión sexual en el periodo 2008 y 2011 en Cundinamarca, es mayor en mujeres entre los 20 y los 29 ańos de edad, siendo el primer agente causal $N$. gonorrhoeae seguido de $C$. trachomatis, en éste estudio el $21,4 \%$ de mujeres con títulos de anticuerpos 
IgG contra $C$. trachomatis fueron asintomáticas (14). El grupo control presentó porcentajes de pacientes positivas a Ig G contra $C$. trachomatis, las personas de éste grupo deben ser estudiadas y se debe evaluar el posible desarrollo de artritis reactiva en un futuro.

La presencia de Ig $\mathrm{G}$ a $C$. trachomatis en una muestra de mujeres entre 18 y 30 años con diagnóstico de artritis, no permitió evidenciar algún tipo de asociación entre la pre infección con $C$. trachomatis y el desarrollo de artritis, se requiere a futuro, implementar nuevos estudios en los cuales se busque el diagnóstico temprano de $C$. trachomatis además de aumentar en tamaño de la muestra.

\section{Referencias}

1. Sociedad española de reumatología. Folleto de la SER: La Artritis reactiva [Internet]. Available from: http://www.ser. es/pacientes/consultas_recursos/folleto.php. Consultado el 2 septiembre de 2015.

2. Matta, S., Arango, Á. I., \& Visbal, J. Chlamydia trachomatis: aspectos microbiológicos, clínicos y epidemiológicos. MVZ -CORDOBA Colombia. 2001; Vol. 6(002):87-96.

3. Hernández DMP, Callejas NR, Rodríguez CM, Morejón JAG, Cuellar IMH, Mantecón AML, et al. Artritis Reumatoide: beneficios clínicos observados en pacientes tratados con anticuerpo monoclonal Itolizumab. (T1h mAB), 2 años después de recibir tratamiento. Revista Cubana de Reumatologia. 2011;13(17 y 18).

4. Quiceno JM, Vinaccia S. Artritis reumatoide: consideraciones psicobiológicas. Divers Perspect Psicol. 2011;Vol. 7(N¹):pp. 27-41.

5. Vinaccia S, Quiceno JM, Remor E. Resilience, illness perception of disease, beliefs and spiritual-religious coping in relation to the health-related quality of life in chronic colombian patients. Anales de Psicologia. 2012; Vol. 28(N² (mayo)):pp.366-377.

6. García Carballo MM, Miraflores Carpio JL, García Parejo Y. Artritis reactiva: a proposito de un caso. Medifam, 2001, vol. 11, no 8, p. 92-94.

7. Molina AT, Mora OU. Síndrome de Reiter en la infancia. Reporte de un caso. Revista Electrónica de las Ciencias Médicas en Cienfuegos. 2011;Vol. 9(4).

8. Wu IB, Schwartz RA. Reiter's syndrome: the classic triad and more. J Am Acad Dermatol. 2008 Jul;59(1):113-21.
9. Zarco Montejo P. Diagnosis and treatment of Chlamydia-induced reactive arthritis. Reumatol Clin. 2012 Mar;8 Suppl 1:S20-25.

10. Arráiz N, Marcucci R, Colina S, Reyes F, Rondón N, Bermúdez V, et al. Chlamydia trachomatis infection in females consulting health centres in Maracaibo, Venezuela. Rev Salud Pública Bogotá Colomb. 2008 Oct;10(4):615-24.

11. Mendoza G, Rocha A, Guerra A, Ramírez M, Gonzalez A, Gomez J, Nava A. Artritis reumatoide y dislipidemias. El Residente, 2013, vol. 8, No 1, p. 12-22.

12.Páez, L. C. C., et al. (2015). “Comparación del cultivo celular de HeLa y HEp-2: Perspectivas de estudios con Chlamydia trachomatis.” Nova 13(23). 